\title{
Topography-Based Rigid Gas Permeable Lens Fitting in Normal Corneas: The Relevance of Eyelid and Tear Film Attributes
}

\author{
Genís Cardona, Ph.D., and Roser Isern, M.Sc.
}

\begin{abstract}
Objectives: The aim of this study was to investigate the influence of eyelid and tear film attributes on the ability of a topography-based contact lensfitting module to correctly predict rigid gas permeable (RGP) contact lens parameters leading to successful in situ final lens fitting in normal corneas. Methods: A total of 28 healthy eyes with spherical refraction ranging from -2.00 to $-6.00 \mathrm{D}$, refractive and anterior corneal astigmatism $<-0.75 \mathrm{D}$ and smooth keratometric mires were fitted with diagnostic trial tricurve RGP lenses as determined through simulated fluorescein pattern analysis. Modifications in the back optic zone radius (BOZR) and total diameter (TD) were implemented to the suggested parameters until a satisfactory fit was observed, whereupon the differences from the suggested parameters were recorded. Eyelid position, upper lid tonus, blinking completeness, tear film volume, and quality and corneal eccentricity were evaluated, and their relationship with the number of required parameter modifications was explored.

Results: Eyelid position and tear meniscus height presented a statistically significant association with the number of parameter changes between simulated and final in situ fittings. In addition, although eyelid position influenced both BOZR $(\mathrm{rho}=0.436 ; P=0.020)$ and TD $(\mathrm{rho}=-0.398$; $P=0.036$ ), tear meniscus height only prompted TD modifications ( $\mathrm{rho}=0.435$; $P=0.021$ ). No additional associations were discovered and, on examining the direction of the modifications, no clear tendency could be detected.

Conclusions: Eyelid position and tear meniscus height attributes, not taken into consideration by topography-based lens fitting software, influence RGP lens parameter selection.
\end{abstract}

Key Words: Computer-assisted lens fitting-Contact lenses-Corneal topography_Rigid gas permeable lenses-Simulated fluorescein patterns.

(Eye \& Contact Lens 2011;37: 359-364)

W ith the advent of highly oxygen permeable silicone-hydrogel materials, the number of rigid gas permeable (RGP) contact lens fits has experienced a gradual decline in recent years. Indeed, although in $199139 \%$ of new fits in the United Kingdom were RGP lenses, ${ }^{1}$ the latest annual surveys have revealed RGP prescriptions to have fallen as low as $11 \%$ to $13 \%{ }^{2,3}$ with a reported larger proportion of RGP refits over new fits. ${ }^{4}$ However, RGP contact lenses are still considered the best option for patients with irregular

From the Research Group of Centre Universitari de la Visió (GRCUV) (G.C., R.I.), Optometry Department, Technical University of Catalonia, Catalonia, Spain.

The authors have no funding or conflicts of interest to disclose.

Address correspondence and reprint requests to Genís Cardona, Ph.D, Escola Universitària d'Òptica i Optometria de Terrassa, Violinista Vellsolà, 37, E08222 Terrassa, Catalonia, Spain; e-mail: gcardona@oo.upc.edu, genis. cardona@gmail.com

Accepted August 15, 2011.

DOI: $10.1097 /$ ICL.0b013e318232e431 corneal astigmatism (as in keratoconus, keratoplasty, or after unsuccessful refractive surgery), in orthokeratology, ${ }^{5}$ and as translational bifocal contact lenses, an attractive and optically superior alternative to simultaneous vision for the correction of presbyopia. In addition, RGP lens materials have been associated with a higher resistance to deposits than hydrogel lenses, fewer solution reactions and toxic allergies, ${ }^{6}$ and a low incidence of microbial keratitis. ${ }^{7}$

Initial patient discomfort and increased fitting complexity, often resulting in prolonged chair times and in a larger number of diagnostic trial lenses, have been described as manifest disadvantages of RGP lenses, compared with hydrogel or silicone-hydrogel lenses. ${ }^{8}$ Although new RGP lens designs have been introduced to increase patient comfort, fitting difficulties are still perceived as an RGP lens deterrent by some practitioners.

Computer-assisted RGP contact lens fitting, which is based on second-generation computerized videokeratoscopes, employs corneal topography to determine the optimal RGP lens parameters for each particular patient. Practitioners are allowed access to the existing, constantly updating RGP lens database to modify the suggested parameters until acceptable simulated fluorescein patterns are observed, whereupon the patient is fitted with the first pair of diagnostic trial lenses.

The application of topography-based RGP lens fitting software has been documented on keratoconus, ${ }^{9-12}$ after laser in situ keratomileusis (LASIK) ${ }^{13}$ and keratoplasty, ${ }^{9,14}$ on corneal scarring and on other irregularly shaped corneal surfaces. ${ }^{9}$ Many of these studies report a higher degree of success and efficiency with this procedure than with the traditional method exclusively based on diagnostic trial lenses. Their findings not only acknowledge that central keratometric readings alone are insufficient to fully describe an irregularly shaped corneal surface but also that a successful fitting should complement the assessment of static, simulated fluorescein patterns with the clinical examination of lens movement and centration, and in situ fluorescein patterns, on the day lenses are first dispensed and after they are worn over a period of time. A reduction in fitting time and in the number of trial lenses, increasing patient comfort and moderating the risk of abrasion or cross infection, has been mentioned as an additional advantage of topography-assisted RGP lens fitting in these conditions. ${ }^{11}$

Normal corneas have also been found to profit from corneal topography and computer-assisted lens parameters determination. In effect, a knowledge of midperipheral and peripheral radii was able to explain many of the changes in lens parameters between topography-assisted RGP lens fitting and fitting based on predictions from central keratometric readings. ${ }^{8,15}$ Similar benefits were described when evaluating soft toric contact lens fitting in 
patients with high or irregular peripheral astigmatism. ${ }^{16}$ Consequently, success rates of topography-based RGP lenses fitting on normal corneas have been found to approximate $80 \%,{ }^{17,18}$ although some authors failed to discover any differences between traditional (empirical) and computer-assisted lens fitting. ${ }^{18}$ Interestingly, however, topography-based fitting was reported to require on average $51.4 \%$ less time than required by traditional fitting. ${ }^{18}$

Experienced contact lens practitioners are aware that the dynamics of in situ RGP lenses are influenced by factors such as eyelid position and tension, blinking, and tear film characteristics. As far as we know, these factors are not taken into consideration by any topography-based contact lens fitting software. Indeed, it is not uncommon for simulated fluorescein patterns to be insufficient to correctly identify the most appropriate final RGP lens parameters for a particular patient, thus requiring one or more changes (in radius, diameter) to be implemented before a satisfactory fit is achieved.

The aim of this study was to explore the influence of eyelid position and tension, blinking completeness and tear film quality and volume on the ability of an RGP lens fitting software to determine optimal lens parameters based on acceptable simulated fluorescein patterns. Therefore, we evaluated the number of changes in back optic zone radius (BOZR) and total diameter (TD) that needed to be implemented over the contact lens parameters suggested by the software fitting (SF) to achieve an optimal in situ fitting (OF), as assessed by an independent experienced contact lens practitioner. The relationship between the number and direction of the changes in BOZR and TD and eyelid, blinking, and tear film attributes, and corneal eccentricity, was investigated.

\section{MATERIALS AND METHODS}

\section{Participants}

Twenty-eight patients attending an optometric practice located in the city of La Seu d'Urgell (Spain) were recruited for this study, which took place between January and November 2010. All the patients were new contact lens wearers with best-corrected distance binocular visual acuity 1 or greater, were free of amblyopia and binocular vision anomalies, and had an adequate tear film (mean self-reported Ocular Surface Disease Index score of 14.12; $\mathrm{SD}=3.93$ ). All the eyes had a vertex-compensated spherical prescription between -2.00 and $-6.00 \mathrm{D}$ (inclusive), spectacle and anterior corneal cylinder $<-0.75 \mathrm{DC}$ and smooth keratometric mires. Exclusion criteria were existing or previous ocular pathologic conditions, corneal staining, ongoing ocular treatment, and a history of ocular or refractive surgery. In addition, patients with poor motivation or unwillingness to try out RGP lenses and those experiencing high initial levels of discomfort or RGP lens intolerance were excluded from the study.

All the participants provided written informed consent after the nature of the study was explained to them. The study was conducted in accord with the Declaration of Helsinki tenets of 1975 (as revised in Tokyo in 2004). The study received the approval of an Institutional Review Board (Technical University of Catalonia).

\section{Instrumentation and Rigid Gas Permeable Contact Lenses}

The Oculus Easygraph (Oculus Optikgeräte GmbH, Wetzlar, Germany) uses Placido reflective rings to determine corneal topography. It includes a contact lens fitting software
(Hecht Contactlinsen GmbH, Baden-Württemberg, Germany) with a database of aspheric and multicurve lens designs from several manufacturers, which may be updated when new lens designs become available (Fig. 1). This software suggests initial RGP lens parameters based on central keratometric radii and average eccentricity values at $30^{\circ}$. In addition, although the contact lens fitting software aims for an alignment fit, contact lens practitioners may further alter the diverse contact lens parameters to obtain a simulated fluorescein fit matching their particular goals or fitting philosophy. For the purposes of this study, the default settings of the contact lens fitting software were initially untouched.

All the eyes were fitted with the same Boston XO (Bausch \& Lomb Inc., Rochester, NY) tricurve RGP lens design and material. Table 1 summarizes lens specifications.

\section{Procedures}

At the beginning of the study, the subjects were given a comprehensive ocular examination to determine ocular health and to define the final sample by ensuring that they met the inclusion/exclusion criteria described above. Ocular parameters were measured, including corneal topography, horizontal visible iris diameter, and mesopic pupil diameter (with the infrared Colvard pupillometer, Oasis Medical). Eyelid position and blinking completeness were recorded with a digital video camera set at 25 frames per second and mounted on the slitlamp. The subjects were asked to maintain a primary gaze position fixation (straight ahead at a distant small fixation light) and to blink normally for $1 \mathrm{~min}$, while being observed under diffuse low illumination and low magnification. Eyelid position with reference to the cornea was evaluated by superimposing a semitransparent clock face over the visible iris (Fig. 2) and graded from 1, describing eyes in which the upper lid margin crossed the visible iris at 9 and $3 \mathrm{o}^{\prime}$ clock, to 5, which corresponded to an upper lid located above the superior corneal limbus. The tonus in the upper lid was assessed during eyelid eversion for examination and classified as loose, average, or tight (grades 1-3). A complete blink was defined by a downward movement of the upper eyelid covering more than $75 \%$ of the cornea, whereupon blinking was classified as incomplete when more than half of the recorded eyeblinks were incomplete. Tear film stability and volume were evaluated with the break-up time (BUT) ${ }^{19}$ and tear meniscus height, ${ }^{20}$ respectively, in accordance with published literature.

The Oculus Easygraph contact lens software was employed to determine initial default BOZR and TD, at which point the simulated fluorescein pattern was assessed and the parameters fine tuned until a mild apical clearance, midperipheral alignment, and acceptable edge clearance was observed. If necessary, simulated lenses were manually repositioned to ascertain good corneal centration.

Once the desired SF was obtained, a diagnostic trial lens of the same parameters was fitted to the patient and allowed to settle for a period of $20 \mathrm{~min}$, after which the fitting was assessed by a different experienced contact lens practitioner, unaware of the goals of the study. Fluorescein patterns, lens movement, and centration were evaluated and, when considered unsatisfactory, the appropriate parameter changes in BOZR and TD were implemented until an OF was achieved. Finally, the fitting was reassessed 3 days later, thus defining the final OF lens parameters to be compared with those determined during SF. 


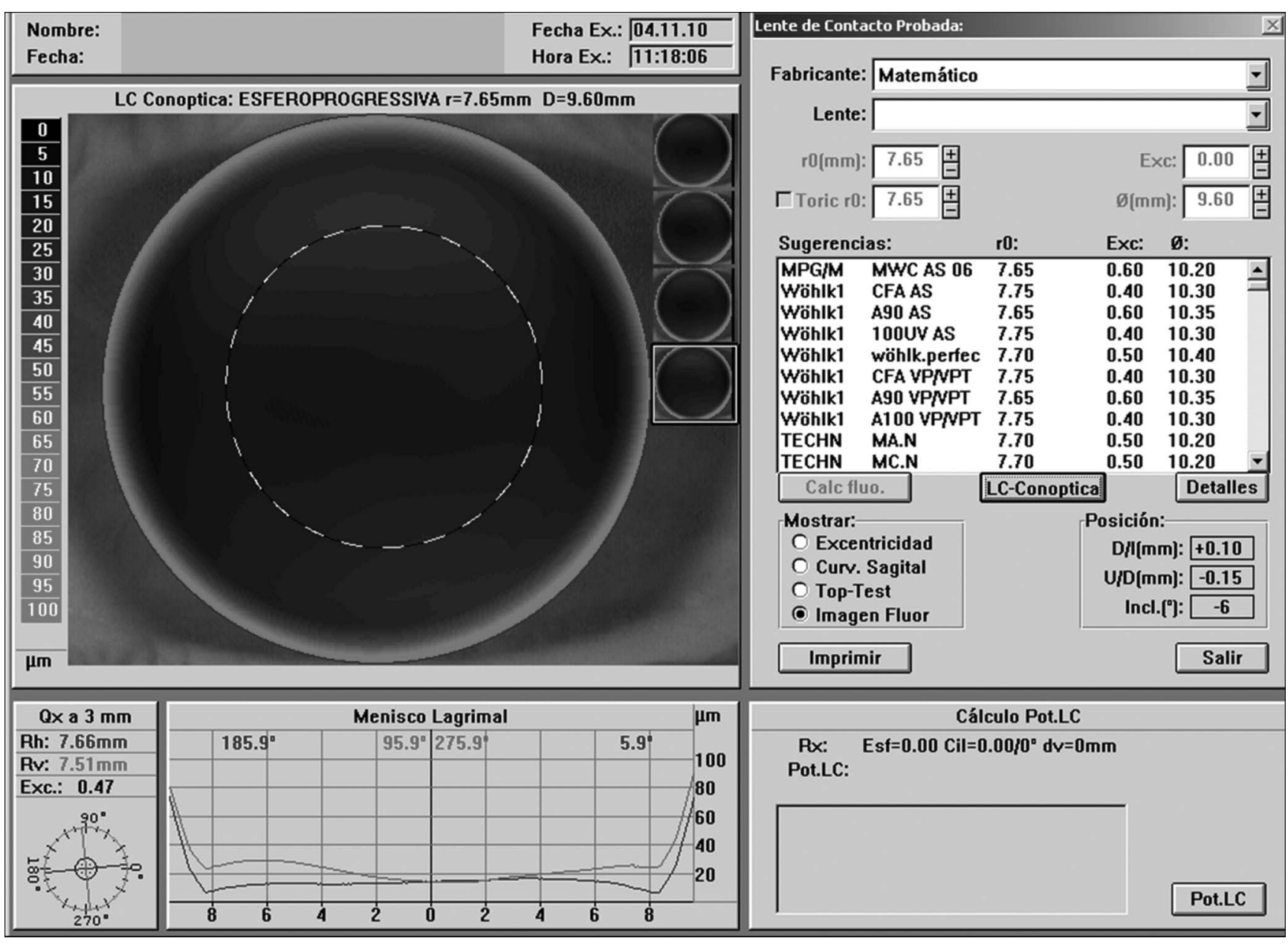

FIG. 1. Screen capture of the Oculus Easygraph contact lens fitting software showing a typical simulated fluorescein pattern of a tricurve rigid gas permeable (RGP) contact lens.

Only BOZR and TD parameter changes were taken into consideration for the purposes of this study. Therefore, modifications in lens power related or not to changes in BOZR were not recorded. Similarly, no stand-alone modifications to peripheral curves or back optic zone diameter (BOZD) were introduced.

\section{Data Analysis}

Statistical analysis of the data was performed with the SPSS software 17.0 for Windows. Sample size was determined by considering a Cohen $d$ effect size of 0.8 and an alpha level of 0.05 ,

TABLE 1. Summary of the Boston XO RGP Contact Lens Specifications

\begin{tabular}{ll}
\hline Material generic name & \multicolumn{1}{c}{ Hexafocon A } \\
\hline Material type & Fluoro silicone acrylate \\
Dk (ISO/Fatt) & 100 \\
Refractive index & 1.415 \\
Modulus & $1,500 \mathrm{MPa}$ \\
Design & Spherical \\
Back optic zone radius & 5.00 to $9.00 \mathrm{~mm}$ in 0.05 increments \\
& (smaller increments are available) \\
Total diameter & 7.00 to $11.50 \mathrm{~mm}$ in 0.20 increments \\
& (smaller increments are available) \\
Back vertex power & -20.00 to $+20.00 \mathrm{D}$ in 0.25 increments \\
\end{tabular}

RGP, rigid gas permeable. resulting in a recommended sample size of approximately 26 . Nonparametric statistical tests were employed throughout the study because data were either ordinal in nature or failed to follow a normal distribution, as examined with the Kolmogorov-Smirnov

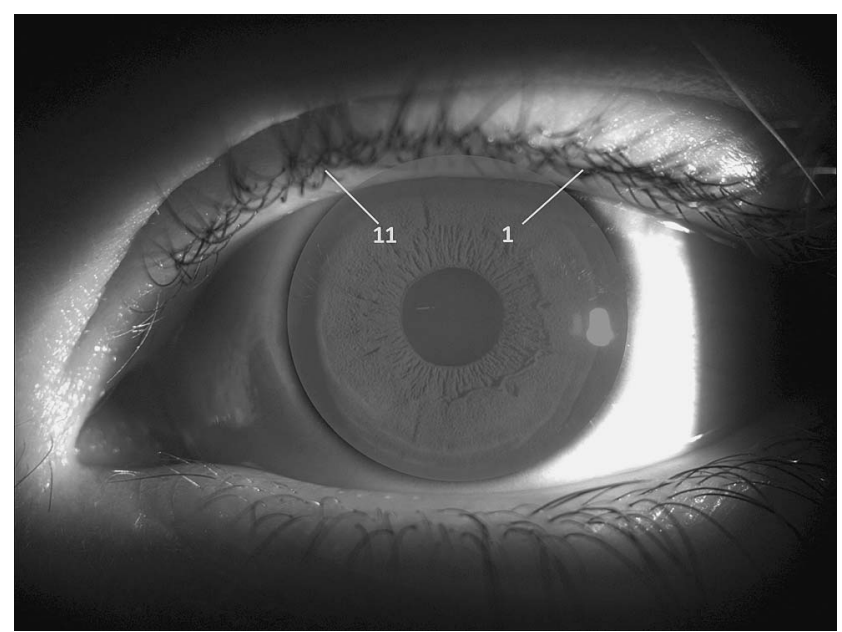

FIG. 2. Evaluation of the upper lid position with reference to the cornea. 
test. Within the same patient, eyes could not be considered to be truly independent. Therefore, data from one eye only were randomly selected for statistical analysis.

To compare SF with OF, BOZR and TD differences between both fittings were scored in open scales by allocating 1 point for each 1 step modification in BOZR $(0.05 \mathrm{~mm})$ and TD $(0.20 \mathrm{~mm})$, respectively. The direction of all changes (steeper or flatter BOZR; larger or smaller TD) was also registered. The Spearman rho correlation test was employed to evaluate the relationship between the number of changes in BOZR and TD and the position of the eyelids with reference to the cornea, upper lid tonus, tear film quality, and volume and corneal eccentricity. The Mann-Whitney test was used to evaluate differences between complete and incomplete blinking in the number of parameter changes. A $P$ value of 0.05 or less was considered to denote statistical significance throughout the study.

\section{RESULTS}

The age of participants ranged between 15 and 56 years (mean=39.9 years; $\mathrm{SD}=14.1$ years). Nineteen subjects were female. Ocular refraction, which as per inclusion criteria ranged between -2.00 and $-6.00 \mathrm{D}$, was evenly distributed among the eyes under study. Central keratometric readings and corneal eccentricity were within the limits of normality.

Table 2 provides a summary of the most relevant measured ocular parameters and tear film characteristics of the present sample. Eyelid position was mostly grade 2 (2-10 o' clock) and grade 3 (1-11 o'clock), and upper lid tonus showed a tendency toward grade 3 , that is, a tight upper eyelid. A moderate percentage of patients demonstrated incomplete eyelid closure on blinking.

Changes in BOZR and TD between SF and OF are shown in Table 3. Although $42.86 \%$ and $75 \%$ of fittings did not require any change in BOZR and TD, respectively, only $28.57 \%$ of simulated fittings were judged as being completely satisfactory without any additional parameter modification. Of the remaining eyes, most fittings were considered acceptable after $1(25 \%)$ or $2(21.43 \%)$ modifications in BOZR, that is, an overall change of 0.05 or $0.10 \mathrm{~mm}$, respectively, or 1 modification in TD $(21.43 \%)$, equivalent

TABLE 2. Ocular Parameters and Tear Film Characteristics of the Study Sample

\begin{tabular}{ll}
\hline & $\mathrm{n}=28$ (Eyes) \\
\hline Age (mean \pm SD) & $39.9 \pm 14.1 \mathrm{yrs}$ \\
Corneal flattest meridian (mean \pm SD) & $7.85 \pm 0.26 \mathrm{~mm}$ \\
Corneal eccentricity (mean \pm SD) & $0.41 \pm 0.17$ \\
Horizontal visible iris diameter (mean \pm SD) & $12.1 \pm 0.3 \mathrm{~mm}$ \\
Mesopic pupil diameter (mean \pm SD) & $5.43 \pm 0.62 \mathrm{~mm}$ \\
Position of the upper eyelid & \\
Grade 1 (position 3-9 o' clock) & $10.71 \%$ \\
Grade 2 (position 2-10 o'clock) & $39.29 \%$ \\
Grade 3 (position 1-11 o'clock) & $32.14 \%$ \\
Grade 4 (position tangent to superior limbus) & $14.29 \%$ \\
Grade 5 (position over superior limbus) & $3.37 \%$ \\
Tonus of the upper eyelid & \\
Grade 1 (loose) & $25 \%$ \\
Grade 2 (average) & $28.57 \%$ \\
Grade 3 (tight) & $46.43 \%$ \\
Completeness of blinking & \\
Complete & $64.3 \%$ \\
Incomplete & $35.7 \%$ \\
Tear meniscus height (mean \pm SD) & $0.4 \pm 0.1 \mathrm{~mm}$ \\
Break up time (mean \pm SD) & $9.8 \pm 1.7 \mathrm{~s}$ \\
\hline
\end{tabular}

TABLE 3. Changes in BOZR and TD and Total Number of Changes Between Simulated and Optimal in Situ RGP Contact Lens Fitting ${ }^{a}$

\begin{tabular}{lccc}
\hline Number of changes & ${\text { BOZR }(\%)^{b}}^{b}$ & TD $(\%)^{b}$ & ${\text { Total }(\%)^{b}}^{b}$ \\
\hline 0 & 42.86 & 75 & 28.57 \\
1 & 25 & 21.43 & 32.14 \\
2 & 21.43 & 3.57 & 21.43 \\
3 & 7.14 & 0 & 14.29 \\
4 & 3.57 & 0 & 3.57 \\
\hline
\end{tabular}

${ }^{a}$ One point was allocated for each 1-step modification in BOZR $(0.05 \mathrm{~mm})$ and TD $(0.20 \mathrm{~mm})$, respectively.

${ }^{b}$ Percentages represent the number of cases.

BOZR, back optic zone radius; TD, total diameter.

to a $0.20-\mathrm{mm}$ change. Only 3 fittings (10.71\%) required a simultaneous modification of both BOZR and TD.

Spearman rho correlation analysis between eyelid position and the number of parameter modifications revealed statistically significant, albeit weak associations for both BOZR ( $\mathrm{rho}=0.436$; $P=0.020$ ) (Fig. 3) and TD (rho $=-0.398 ; P=0.036$ ) (Fig. 4), that is, eyes with smaller palpebral apertures (grades 1 and 2 of eyelid position with reference to the cornea) required a smaller number of modifications in BOZR, but more changes in lens TD, than did eyes with eyelid position graded as 3,4 , or 5 . It may be noted that, as shown in the scatter plots, the clumping of data in certain cells (grades 2 and 3 of eye lid position) probably prevented exposing stronger associations between eyelid position and number of parameter modifications. Furthermore, on examining the direction of these modifications, no clear tendency could be detected. Table 4 shows lid position in terms of fitting success, that is, those cases where no changes in BOZR or TD were required. Although lid position was not found to influence fitting success when defined as no changes in BOZR, a statistically significant difference was encountered between lid positions 2 and 3 in the number of patients not requiring any change in $\mathrm{TD}(P=0.014)$, with a larger number of patients presenting large palpebral apertures. Upper eyelid tonus and parameter modifications did not disclose any statistically

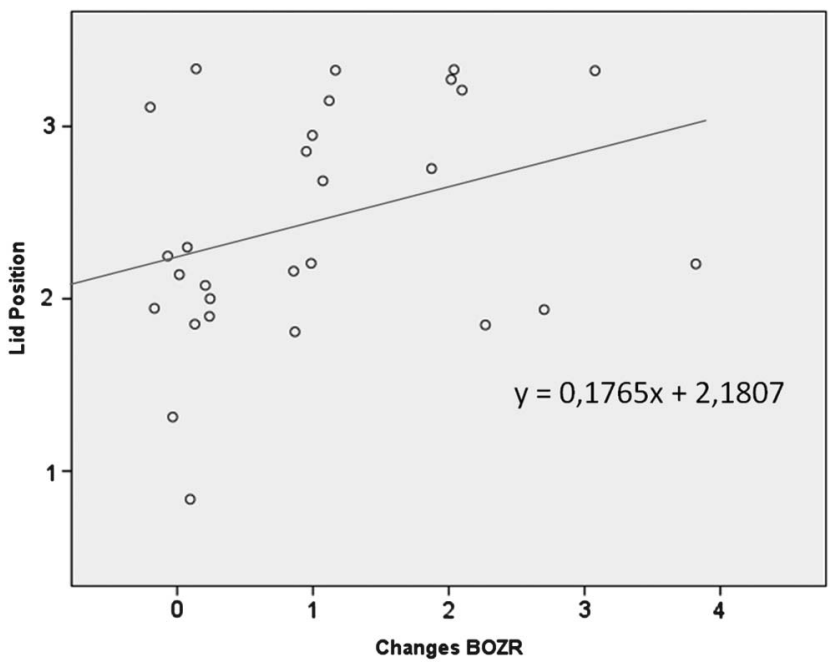

FIG. 3. View of the changes in back optic zone radius versus lid position (as graded from 1 to 5: lower numbers represent a lower eyelid position). 


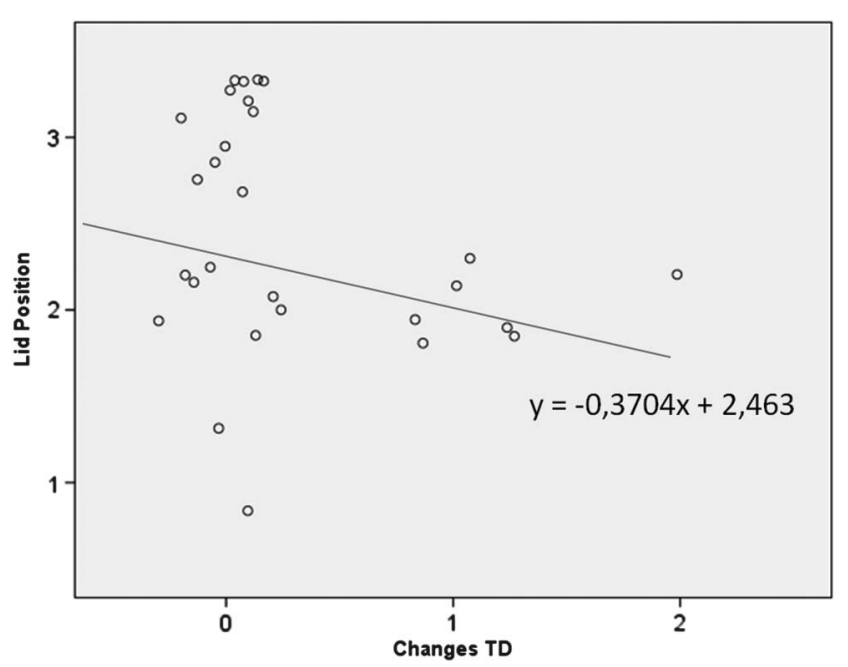

FIG. 4. View of the changes in total diameter versus lid position (as graded from 1 to 5 : lower numbers represent a lower eyelid position).

significant relationship. Similarly, the Mann-Whitney test failed to expose any difference between complete and incomplete blinking in the number of BOZR or TD parameter changes.

Tear film characteristics were found to exhibit an interesting influence over the recorded differences between SF and OF. Thus, although no significant association could be discerned between BUT and the number of parameter modifications, tear meniscus height displayed a statistically significant association with the number of modifications in TD (rho $=0.435 ; P=0.021)$ but not with changes in BOZR ( $\mathrm{rho}=-0.116 ; P=0.556$ ). These findings suggest that an increase in tear film volume results in more TD modifications between SF and OF, and, albeit the results failed to reach statistical significance, a certain trend was observed in which smaller diameters were required to achieve $\mathrm{OF}$ in those eyes with a larger volume of tears. Incidentally, tear meniscus height and BUT were found to display a moderate positive correlation ( $r h o=0.442 ; P=0.019$ ).

Finally, no statistically significant association was encountered between corneal eccentricity and the number of parameter modifications, in either BOZR or TD.

\section{DISCUSSION}

This study aimed at exploring the influence of such factors as eyelid position, upper lid tonus, blinking completeness, and tear film characteristics on the ability of a topography-based contact lens-fitting module to predict simulated RGP lens parameters leading to a satisfactory in situ fitting. The working hypotheses of this investigation was that, because corneal topography, per

TABLE 4. Lid Position in Patients not Requiring any Change in BOZR or TD

\begin{tabular}{lccc}
\hline Parameter & Lid position $1^{a}$ & Lid position $2^{a}$ & ${\text { Lid position } 3^{a}}^{a}$ \\
\hline BOZR & $2 / 11$ & $8 / 11$ & $1 / 11$ \\
TD & $2 / 22$ & $7 / 22$ & $13 / 22$ \\
\hline
\end{tabular}

${ }^{a}$ In the number of cases over total.

BOZR, back optic zone radius; TD, total diameter. definition, does not include information about eyelid and tear film characteristics, any fitting based solely on topographic data and simulated fluorescein patterns assessment may require fine tuning to be considered acceptable when transferred to diagnostic trial lenses.

Previous studies have documented the usefulness of contact lensfitting modules when assisting RGP lens fitting in irregular corneal shapes such as keratoconus, keratoplasty and irregular central or peripheral astigmatism..$^{9-14}$ Other authors have noted the benefits of possessing topographic data from the periphery of the cornea to reduce both chair time and number of diagnostic fitting lenses, even in normal corneas. ${ }^{8,15-18}$ Interestingly, to the best of our knowledge, the factors contributing to discrepancies between simulated and in situ RGP lens fittings have not been explored in detail.

The present findings, with only $28.57 \%$ of simulated fittings not requiring any further parameter modifications, disclose a lower rate of success than that previously reported for normal corneas. However, these discrepancies may be explained by the lack of consensus regarding what should constitute a parameter change. Indeed, although we defined a change as a modification of $0.05 \mathrm{~mm}$ in BOZR or of $0.20 \mathrm{~mm}$ in TD, previous researchers recorded a change as a modification equal or greater than $0.10 \mathrm{~mm}$ in BOZR, a lens power change greater than $0.50 \mathrm{D}$, or a change in TD greater than $0.20 \mathrm{~mm} \cdot{ }^{10,18,21}$ Moreover, these authors did not report the actual number of changes (or steps) that were introduced within each parameter but only the need to implement a modification in one or various lens parameters. By following this reasoning, although 3 changes in this study may imply a modification in BOZR of $0.15 \mathrm{~mm}$, the same 3 changes in previous works may refer to a relatively uncommon simultaneous alteration in BOZR, TD, and lens power. In consequence, the lower degree of success disclosed here is probably not unexpected. Similarly, it was not surprising that, of all parameter changes, the most frequent was a modification in BOZR, in agreement with previous research. ${ }^{10,18}$ Furthermore, it may be noted that we opted for not including changes in BOZD in our study. Albeit BOZD may be considered as relevant as BOZR when determining sagittal depth, as no stand-alone changes were introduced in peripheral curve radii and width, BOZD may be safely interpreted as a dependent variable of both BOZR and TD.

Data analysis revealed that, of the factors under investigation, only eyelid position and tear meniscus height presented a statistically significant association with the number of parameter changes between SF and OF, although these associations were not strong. In addition, although eyelid position influenced both BOZR and TD, tear meniscus height only prompted TD modifications. It may be speculated whether the lack of statistically significant associations between eyelid tonus and blink completeness could be explained by the intrinsic limitations of the scales on which these factors were measured.

Experienced contact lens practitioners are aware of the importance of selecting the appropriate RGP lens TD to maximize eyelid/lens interaction. Indeed, if the upper lid is positioned at or above the superior limbus (grades 4 and 5), it may be preferable to prescribe a smaller TD lens, with a steeper BOZR, to achieve good centration. On the other hand, an intrapalpebral fitting relationship may benefit from a larger TD, to take advantage of eyelid mechanics in lens centration and movement. ${ }^{22}$ The fact that our findings described a larger number of modifications in TD, and less in BOZR, in those eyes with intrapalpebral contact lens fitting relationship may account for the major relevance of fine tuning lens 
TD when eyelid/lens interaction is critical. The same eyelid/lens relationship may help explain the absence of any clear tendency on examining the direction of the implemented modifications, because an optimal intrapalpebral fitting may require an increase in TD (and BOZR), whereas the opposite may be needed to achieve a satisfactory interpalpebral or lid-free fitting. However, it must be acknowledged that a larger sample size would be required to investigate this assumption. Currently, RGP lenses are not the first choice for new wearers with low to moderate myopia, without significant astigmatism and without corneal irregularities (or other complications, such as post-LASIK corneal ectasia), ${ }^{3}$ thus defining patient recruitment as one of the main difficulties of this study.

The influence of tear volume (as measured with tear meniscus height) over TD may be explained by the assumption that larger lenses attract a higher volume of tears to the area underneath the lens, leaving a lower amount of tear volume available on the ocular surface. In effect, because of capillary attraction, when an RGP lens is inserted in the eye, an additional tear meniscus is formed, surrounding the entire lens edge. It may be speculated whether a larger diameter lens, in association with a higher tear meniscus, would result in a magnification of this effect, with a possible role in the development of 3- and 9-o' clock staining, thus leading to an unacceptable lens fit. In agreement with this hypothesis, and although no statistically significant tendencies were encountered, our findings seem to suggest a general trend toward smaller TD in those eyes with a high tear meniscus.

Interestingly, the fact that no statistically significant association was disclosed between corneal eccentricity and the number of BOZR or TD parameter modifications may imply that the ability of our software to determine lens parameters was similar for all corneas. This conjecture, however, must be approached with caution, as our study sample included normal corneas with a relatively narrow range of eccentricities. In addition, it may be interesting to note that the parameters for simulated contact lenses are determined through topography-based algorithms, specific for each topographer and software package and, therefore, slight discrepancies between instruments and contact lens fitting modules may occur.

In conclusion, our findings reveal that lid position, and to a lesser extent, tear meniscus height, are determinant factors in explaining the differences between computer-simulated lens predictions and in situ fittings, in negative spherical RGP lenses of low to moderate power and normal corneas. Although corneal topography is a valuable tool in proposing the initial diagnostic lenses, eyelid position may not be inferred from topographic measurements, because patients are often instructed to open their eyes as much as possible to avoid unwanted anatomic shadows or loss of peripheral data, which must then be extrapolated by software algorithms. All RGP lens fittings must therefore be accurately examined by an experienced practitioner to assess the need to modify any or all lens parameters to achieve a satisfactory final lens fitting, in terms of both quality of vision and ocular surface physiology. In view of the present findings, a large scale, multicenter study, including a variety of lens powers (negative and positive, spherical, and toric) and designs (aspheric, multicurve), may be required to verify the influence of eyelid position and tear volume. An ideal outcome of this future study would result in next generation topography-based contact lens-fitting modules allowing practitioners to upload eyelid position and tear volume attributes to their software to obtain more accurate predictions of simulated RGP lens parameters.

\section{REFERENCES}

1. Pearson R. Contact lens fitting in the United Kingdom. Cont Lens Anterior Eye 1998;21:147.

2. Gill FR, Murphy PJ, Purslow C. A survey of practitioner attitudes to the fitting of rigid gas permeable lenses. Ophthal Physiol Opt 2010;30:731-739.

3. Efron N, Morgan PB, Helland $\mathrm{M}$, et al. International rigid contact lens prescribing. Cont Lens Anterior Eye 2010;33:141-143.

4. Morgan PB. Trends in contact lens prescribing 2010. Optician 2010;239: 34-35.

5. Villa-Collar C, González-Méijome JM, Queirós A, et al. Short-term corneal response to corneal refractive therapy for different refractive targets. Cornea 2009;28:311-316.

6. Phillips A. Rigid gas permeable contact lens fitting. In: Phillips A, Stone J, eds. Contact Lenses, 3rd ed. London, United Kingdom, Butterworth, 1989, pp 333-381.

7. Morgan PB, Efron N, Hill EA, et al. Incidence of keratitis of varying severity among contact lens wearers. Br J Ophthalmol 2005;89:430-436.

8. van der Worp E, de Brabander J, Lubberman B, et al. Optimising RGP lens fitting in normal eyes using 3D topographic data. Cont Lens Anterior Eye 2002;25:95-99.

9. Nosch DS, Ong GL, Mavrikakis I, et al. The application of a computerised videokeratography (CVK) based contact lens fitting software programme on irregularly shaped corneal surfaces. Cont Lens Anterior Eye 2007;30; 239-248.

10. Donshik PC, Reisner DS, Luistro AE. The use of computerized videokeratography as an aid in fitting rigid gas permeable contact lenses. $\operatorname{Tr} \mathrm{Am}$ Ophth Soc 1996;94:135-145

11. Bhatoa NJ, Hau S, Ehrlich DP. A comparison of a topography-based rigid gas permeable contact lens design with a conventionally fitted lens in patients with keratoconus. Cont Lens Anterior Eye 2010;33:128-135.

12. Sorbara L, Dalton $\mathrm{K}$. The use of video-keratoscopy in predicting contact lens parameters for keratoconic fitting. Cont Lens Anterior Eye 2010;33:112-118.

13. Szczotka-Flynn LB, Jani BR. Comparison of axial and tangential topographic algorithms for contact lens fitting after LASIK. Eye Contact Lens 2005;31:257-262.

14. Szczotka LB, Reinhart W. Computerized videokeratoscopy contact lens software for RGP fitting in a bilateral postkeratoplasty patient: A clinical case report. CLAO J 1995;21:52-56.

15. Chan JS, Mandell R, Johnson L, et al. Contact lens base curve prediction from videokeratography. Optom Vis Sci 1998;75:445-449.

16. Szczotka LB, Roberts C, Herderick EE, et al. Quantitative descriptors of corneal topography that influence soft toric contact lens fitting. Cornea 2002; 21:249-255.

17. Jani BR, Szczotka LB. Efficiency and accuracy of two computerized topography software systems for fitting rigid gas permeable contact lenses. CLAO J 2000;26:91-96.

18. Szczotka LB. Clinical evaluation of a topographically based contact lens fitting software. Optom Vis Sci 1997;74:14-19.

19. Lemp MA, Hamill JR. Factors affecting tear film break-up in normal eyes. Arch Ophthalmol 1973;89:103-105.

20. Port MJA, Asaria TS. Assessment of human tear volume. J Br Contact Lens Assoc 1990;13:76-82.

21. Szczotka LB, Capretta DM, Lass JH. Clinical evaluation of a computerized topography software method for fitting rigid gas permeable contact lenses. CLAO J 1994;20:231-236

22. Bennett ES, Weissman EA, eds. Clinical Contact Lens Practice. Philadelphia PA, Lippincott Williams \& Wilkins, 2004. 